\title{
ANÁLISE DO GRAU DE DEPENDÊNCIA E PREDISPOSIÇÃO À ÚLCERA DE PRES- SÃO EM PACIENTES DE HOSPITAL UNIVERSITÁRIO
}

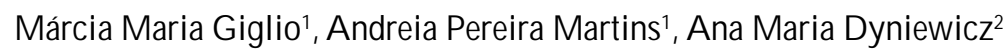

\begin{abstract}
RESUM O: A nalisou-se a predisposição para úlceras de pressão em 23 pacientes de Clínica M édica e 17 de Neurologia. Por meio de observação não participante, aplicou-se nos 40 pacientes um Sistema de Classificação de Pacientes (SCP) e a seguir a Escala de Braden (E.B) entre agosto a outubro de 2005. Obteve-se como resultado: 02 pacientes em cuidados mínimos, 17 em cuidados intermediários, 18 em semi-intensivos e 03 em intensivos. Pela EB, 08 pacientes em risco leve, 10 em risco moderado e 22 com risco alto para o desenvolvimento de úlcera de pressão (UP). D entre os pacientes, 10 foram admitidos com UP, sendo 07 acima de 60 anos e 05 pacientes desenvolveram U P entre 8 e 26 dias de internamento. Os pacientes obtiveram grau de dependência alto e os idosos com maior predisposição e desenvolvimento de UP. H ouve correlação direta entre as condições de trabalho em enfermagem e as necessidades de cuidado.
\end{abstract}

DESCRITORES: Enfermagem; Úlcera de pressão; Cuidados de enfermagem.

\section{ANALYSIS OF DEPENDENCE DEGREE AND PREDISPOSITIONTO PRESSURE ULCERS IN PATIENTSAT A TEACHING HOSPITAL}

ABSTRACT: It was analyzed the predisposition to pressure ulcers in 23 patients in the M edical Clinic and 17 of $\mathrm{N}$ eurology. Through outside observation, a patient classification system was applied in 40 pati ents follow ed by B raden scale, from A ugust to 0 ctober/2005. The result was as follows: 02 patients in simple care, 17 in intermediary care, 18 in semi-intensive care and 3 in intensive care. A ccording to B raden Scale, 8 patients were in mild risk, 10 in moderate risk and 22 in high risk to develop pressure ulcers. A mong the patients, 10 were admitted with pressure ulcer, of those, 7 were over 60 years old, and 05 patients developed pressure ulcer between 8 and 26 days of admittance. They had a high degree of dependence and the elderly had higher predisposition and development of pressure ulcer. There was a straight correlation between nursing working conditions and care needs.

DESCRIPTORS: N ursing; Pressure ulcer; Nursing care.

\section{ANÁLISIS DEL GRADO DE DEPENDENCIA Y PREDISPOSICIÓN A ÚLCERA POR PRESIÓN EN PACIENTES DE HOSPITAL UNIVERSITARIO}

RESUMEN: Fue analizada la predisposición a úlceras por presión en 23 pacientes de Clínica M édica y 17 de $\mathrm{N}$ eurología. Por medio de observación no participante, se aplicó en los 40 pacientes un Sistema de Clasificación de Pacientes (SCP) y, a seguir, la E scala de Braden (EB), entre agosto y octubre de 2005. Se obtuvo como resultados: 02 pacientes en cuidados mínimos; 17 en cuidados intermedios; 18 en semiintensivos; y 03 en intensivos. Por la EB, 08 pacientes en riesgo leve, 10 con riesgo moderado y 22 con riesgo severo para el desarrollo de úlcera por presión (UP). Entre los pacientes, 10 fueron admitidos con UP, siendo 07 con más de 60 años y 05 pacientes desarrollaron U P entre 8 y 26 días de internamiento. L os pacientes obtuvieron grado de dependencia al to y los ancianos presentaron mayor predisposición y desarrollo de UP. Hubo correlación directa entre las condiciones de trabajo en enfermería y las necesidades de cuidado.

DESCRIPTORES: Enfermería; Ú Icera por presión; A tención de enfermería.

\footnotetext{
${ }^{1}$ Enfermeira. M embro do Núcleo de Pesquisa Multiprofissional - NUPEM do Hospital Evangélico de Curitiba - HEC. Faculdade Evangélica do Paraná - FEPAR.

${ }^{2}$ Enfermeira. D outora em Enfermagem. Coordenadora do NUPEM do HEC/FEPAR.
} 


\section{INTRODUÇÃO}

A enfermeira tem buscado promover a assistência de enfermagem ao indivíduo de forma cada vez mais científica e independente de outros profissionais da área de saúde, tanto na abordagem de novas situações como no reestudo de algumas situações em que a prática tem oferecido resultados importantes para a enfermagem, visando a uma maior autonomia ao profissional(1).

Dentre as diversas ações que as enfermeiras realizam nesse sentido, estão a prevenção e o tratamento às Ú Iceras de Pressão (UP), historicamente relacionados aos cuidados prestados aos pacientes internados nas diferentes especial idades inseridas no âmbito hospitalar.

A s UP se constituem em um importante agravamento do quadro clínico de pacientes internados por longos períodos. O correm por uma pressão que é aplicada à pele por um período de tempo maior que a pressão fisiológica de fechamento capilar. Essa pressão prolongada impede o fluxo sanguíneo adequado, reduzindo, assim, a nutrição da região(2). Prolonga a hospitalização, dificulta a recuperação do paciente e aumenta o risco de desenvolvimento de outras complicações, como por exemplo, infecções.

A lém desses fatores prejudiciais, as UP acrescentam ao paciente sofrimento físico e emocional, reduzindo a sua independência e funcionalidade na realização de suas atividades diárias, bem como comprometendo o processo educacional direcionado a sua reabilitação.

Desse modo, a equipe multiprofissional deve estar atenta às UP no sentido de prevenir o seu desenvolvimento e favorecer o tratamento. Certamente poderiam ser evitadas se os profissionais tivessem maior conhecimento sobre as principais características dos pacientes que as desenvolvem e sobre as escalas que avaliam a predisposição ao desenvolvimento das UP, possibilitando, portanto, a prevenção(3).

Este trabalho propõe a utilização do Sistema de Classificação de Pacientes e da Escala de B raden (EB) para identificar a predisposição de pacientes hospitalizados a desenvolverem úlceras de pressão. A EB utiliza parâmetros para mensurar a percepção sensorial, umidade, atividade, mobilidade, nutrição, fricção e cisalhamento(4)

O Sistema de Classificação de Pacientes enumera os cuidados que o paciente necessita de acordo com os critérios: Cuidados Intensivos,
Cuidados Semi-intensivos, Cuidados Intermediários e Cuidados M ínimos ${ }^{(5)}$.

Espera-se com esse estudo contribuir para alertar a equipe de enfermagem sobre os cuidados precoces com a pele, diminuindo o tempo de internamento, as re-internações e o risco de infecções secundárias e, igualmente, aumentar a qualidade da assistência de enfermagem e o conforto do paciente.

\section{BASESTEÓRICAS}

Vários termos têm sido utilizados para definir as UP, como escaras, úlceras de decúbito ou feridas de pressão; no entanto, esta terminologia vem sendo reconhecida internacionalmente, à medida que a pressão é o fator etiológico mais relevante na gênese dessas lesões localizadas na pele e causadas por interrupção do suprimento sanguíneo adequado de uma região. Geralmente são provocadas por pressão, cisalhamento e fricção, ou uma combinação desses três fatores ${ }^{(6-7)}$.

Pressão é o fator externo mais importante, pois quando o tecido mole do corpo é comprimido entre uma saliência óssea e uma superfície dura, ocorre uma pressão maior do que a pressão capilar, resultando em isquemia localizada na região. A resposta fisiológica do organismo é mudar de posição para que a pressão seja redistribuída. Cisalhamento ocorre quando a pele permanece estática e os tecidos subjacentes se movem. Pode vir a deformar e destruir os tecidos e, conseqüentemente, danificar os vasos sangüíneos. Fricção ocorre quando duas superfícies são esfregadas uma na outra. A causa mais comum é deslocar o paciente sobre o leito ao invés de levantáIo. Essa prática remove as camadas superiores de células epiteliais.

Pode-se também destacar a umidade que aumenta 0 efeito da fricção, estando presente na pele do paciente pelo suor excessivo ou incontinência urinária.

Outro fator importante a ser contemplado, é o longo tempo que os indivíduos hospitalizados permanecem deitados. Quando em colchões de espuma com baixa densidade ou macas ou mesas cirúrgicas promovem a exposição de proeminências ósseas. Essa exposição também acontece pela falta de mobilização do paciente, pela equipe de saúde, devido aos mais diversos motivos ${ }^{(7)}$.

M esmo estando freqüentemente sujeito a al guns ou a todos os fatores externos, o corpo humano não 
desenvolve úlceras de pressão, automaticamente. Os principais fatores vêm do próprio paciente. Determinam-se assim os fatores internos ao paciente, que são: estado geral, pois o organismo consegue suportar maior pressão externa quando saudável; diminuição da percepção sensorial, ocasionada por patologias, sedação ou anestésicos, dificultando a percepção de dor ou desconforto, comprometendo assim a capacidade de identificar as regiões do corpo que necessitam de alívio da pressão; idade, pois conforme as pessoas envelhecem, sua pele se torna menos elástica e mais fina, el evando a probabilidade a UP; mobilidade reduzida, afeta a capacidade de aliviar a pressão de modo significativo, predispondo ao cisalhamento e a fricção se o paciente estiver restrito ao leito; estado nutricional reduzido, prejudica a elasticidade da pele ea longo prazo pode levar à anemia e à redução de oxigênio aos tecidos; incontinência urinária, contribui para a maceração da pele pela umidade excessiva a que fica exposta; déficit do suprimento sanguíneo, na periferia reduz a pressão capilar normal e provoca desnutrição nos tecidos ${ }^{(7)}$.

Concomitante aos fatores que predispõem os indivíduos às UP, o processo de aval iação do paciente é complexo e extenso, pois busca identificar, precocemente, a predisposição destes para esse tipo de lesão, podendo assim implementar-se medidas de prevenção específicas ${ }^{(6)}$.

A prevenção é o tópico mais importante no tratamento das UP, visando, principalmente, à diminuição da pressão sobre as saliências ósseas, por meio da mobilização continuada em interval os menores que duas horas, e da utilização de colchões apropriados para este fim. Evita também a umidade cutânea constante, os atritos da pel e com a superfície de contato e previne infecções, melhorando o estado nutricional( ${ }^{(8)}$.

\section{METODOLOGIA}

0 estudo foi desenvolvido em duas clínicas de um hospital universitário de grande porte: Clínica M édica composta de 32 leitos, sendo 14 leitos para pacientes do sexo feminino e 18 leitos para pacientes do sexo masculino; N eurologia Clínica e Cirúrgica com 37 leitos, sendo 15 leitos para pacientes do sexo masculino e 22 para pacientes do sexo feminino. A mbas mantêm 100\% da taxa de ocupação.

0 projeto foi submetido e aprovado sob n. 2181/ 05 no Comitê de Ética em Pesquisa da Sociedade Evangélica Beneficente e todos os pacientes ou seus responsáveis assinaram o Termo de Consentimento Livre e Esclarecido.

Para a coleta dos dados foi utilizado, primeiramente, o Sistema de Classificação de Pacientes (SCP), para categorizar pacientes de acordo com os cuidados de enfermagem em: cuidados intensivos, semiintensivos, intermediários e mínimos. Esta coleta ocorreu entre 12 de agosto a 14 de outubro de 2005, em todos os pacientes internados nas unidades estudadas, até 24 horas após o internamento.

Dentre os pacientes submetidos ao SCP, àqueles classificados em cuidados intermediários, semiintensivos e intensivos foi aplicada a Escala de B raden (EB) até 24 horas após o internamento. A cada 48 horas, a $E B$ foi re-aplicada até que o paciente recebesse alta hospitalar, transferência das unidades, óbito ou desenvolvesse úlcera de pressão(9).

A aplicação destes instrumentos ocorreu por meio da observação não-participante até outubro de 2005. Para anál ise dos dados foram utilizadas análises estatísticas.

\section{APRESENTAÇÃO E ANÁLISE DE RESULTADOS}

Fizeram parte deste estudo 40 pacientes. A idade variou de 19 a 92 anos; houve predomínio de pacientes com idade igual ou acima de 60 anos (23 pacientes) e 23 pacientes pertenciam ao sexo feminino. Em relação ao nível de instrução, 18 pacientes eram analfabetos, 16 pacientes tinham 10 grau incompleto; um com $1^{0}$ grau completo e outros 2 pacientes com $2^{\circ}$ grau completo. Em 3 situações não foi possível coletar este dado.

$\mathrm{Na}$ figura 1 estão os resultados da aplicação do SCP em de 23 pacientes da clínica médica.



Figura 1 - R esultados da aplicação do SCP em Clínica M édica. Curitiba, 2005 
Os pacientes da Clínica M édica receberam a seguinte classificação: 02 pacientes em cuidados mínimos e receberam alta hospitalar sem UP; 10 pacientes em cuidados intermediários, sendo 02 admitidos com UP, 06 receberam al ta hospitalar sem UP, 02 foram a óbito sem UP, 01 não foi avaliado por não querer participar da pesquisa e 01 permaneceu internado sem UP; 09 pacientes em cuidados semiintensivos, sendo 03 admitidos com UP, 2 desenvolveram UP ao longo do internamento, 05 foram a óbito e 02 pacientes receberam alta hospitalar sem UP; 02 pacientes em cuidados intensivos, sendo 01 admitido com UP e evoluiu para óbito sem UP, 01 desenvolveu UP ao longo do internamento.

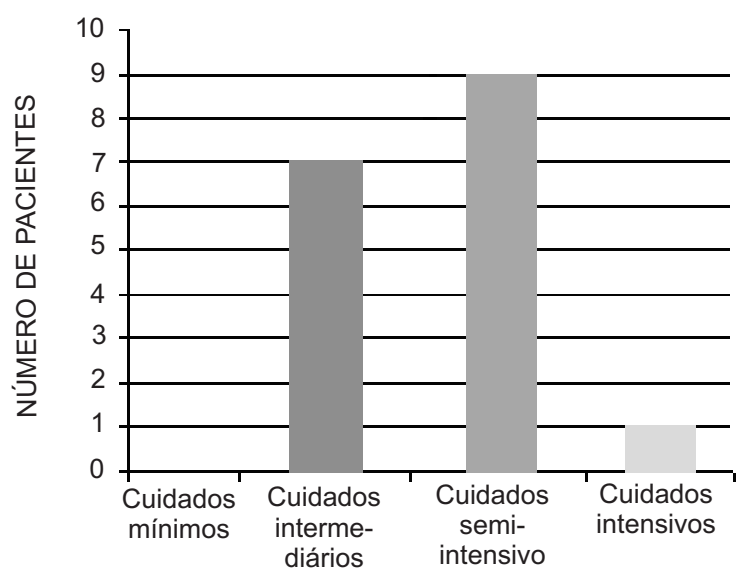

Figura 2 - Resultados da aplicação do SCP em Neurologia Clínica e Cirúrgica. Curitiba, 2005

Os pacientes da N eurologia Clínica e Cirúrgica receberam a seguinte classificação: não houve pacientes na classificação de cuidados mínimos; 07 pacientes receberam a classificação em cuidados intermediários sendo que 01 foi admitido com UP, 02 desenvolveram UP ao longo do internamento, 04 receberam al ta hospitalar sem UP e 01 paciente foi transferido para a UTI sem UP; 09 pacientes em cuidados semi-intensivos, sendo 03 admitidos com UP, 01 permaneceu internado após o término da pesquisa sem UP, 01 recebeu alta hospitalar sem UPe 07 pacientes foram a óbito sem UP; 01 paciente em cuidados intensivos transferido para a UTI sem UP.

Como mostram as Figuras 1 e 2 anteriores, foram classificados em grau de dependência 23 pacientes da Clínica M édica e 17 pacientes da Neurologia Clínica e N eurocirúrgica, totalizando 43 pacientes. Foram excluídos os 02 pacientes em cuidados mínimos da Clínica Médica e incluídos outros dois com grau de dependência maior para completar uma amostra de 40 pacientes.

Os dados do Quadro 1, na página seguinte, mostram os resultados da aplicação da Escala de B raden nesse grupo de pacientes. $\mathrm{Na}$ definição dos escores de risco à úlcera de pressão dos pacientes, levou-se em consideração o mai or número de dias nos níveis de risco. Tomou-se como referência os seguintes níveis: risco leve (escores 15 e 16); risco moderado (escores de 12 a 14) e risco al to para escores iguais ou inferiores a $11^{(10)}$.

Os dados coletados sobre risco à úlcera de pressão mostraram: 08 pacientes em Risco Leve, sendo 06 na Clínica M édica e 04 na N eurologia. Um paciente da Clínica M édica foi admitido com úlcera de pressão em região de calcâneo $D$ e E, em estágio II. Não desenvolveu outras lesões ao longo do internamento.

Em Risco Moderado houve 06 pacientes na Clínica M édica e 02 na N eurologia. D entre esses 10 pacientes 04 apresentavam UP com as seguintes características: a) U m paciente admitido na CM com úlceras de pressão: uma em região trocantérica $D$, em estágio III, infectada com esfacelos; outra em região de tuberosidade isquiática $D$, em estágio II. A inda uma outra em região sacral, em estágio II, em processo de cicatrização, com teci do de granulação, sem esfacelos e áreas de necrose. Esse paciente não desenvolveu outras úlceras durante o internamento. b) Outra paciente, após 12 avaliações, desenvolveu úlceras de pressão em região de extremidade lateral do pé $D$ em estágio I. c) $\mathrm{Na}$ N eurologia, um paciente foi admitido com UP. Não desenvolveu outras ao longo do internamento. d) Outro paciente desenvolveu UP em região de maléolo lateral $D$ em estágio I, após 4 avaliações. A presentava-se comunicativo, sem movimentação e sensibilidade de M M II, al imentandose adequadamente. Sua acompanhante era pouco esclarecida sobre as medidas preventivas para evitar úlceras de pressão.

Em Risco Alto na Clínica M édica havia 10 pacientes: a) 04 foram admitidos com úlcera de pressão. b) 02 desenvolveram UP ao longo do internamento, 01 com UP em região sacral e torácica posterior infectadas, em estágio II. Esteve sem acompanhante. Durantea col eta de dados, observou-se que o paciente sentia-se mais confortável em decúbito lateral $\mathrm{E}$, permanecendo nesta posição a maior parte do tempo. Realizava discreta movimentação de M IE, sem ajuda, fato que, talvez, tenha 
Quadro1 - Resultados da aplicação da E.B em Clínica M édica e N eurologia Clínica e Cirúrgica. Curitiba, 2005

\begin{tabular}{|c|c|c|c|c|c|c|c|c|c|c|c|c|}
\hline \multirow{3}{*}{$\begin{array}{l}\text { Característica } \\
\text { Risco }\end{array}$} & \multirow{2}{*}{\multicolumn{2}{|c|}{$\begin{array}{l}\text { Número de } \\
\text { pacientes }\end{array}$}} & Ad & & \multicolumn{4}{|c|}{ Ao longo do internamento } & \multicolumn{4}{|c|}{ Evolução } \\
\hline & & & \multicolumn{2}{|c|}{$\begin{array}{c}\text { Com úlcera de } \\
\text { pressão }\end{array}$} & \multicolumn{2}{|c|}{$\begin{array}{c}\text { Com úlcera de } \\
\text { pressão }\end{array}$} & \multicolumn{2}{|c|}{$\begin{array}{l}\text { Sem úlcera de } \\
\text { pressão }\end{array}$} & \multicolumn{2}{|c|}{ Alta } & \multicolumn{2}{|c|}{ Óbito } \\
\hline & CM* & $\mathbf{N} * *$ & $\mathbf{C M}$ & $\mathbf{N}$ & $\mathbf{C M}$ & $\mathbf{N}$ & $\mathbf{C M}$ & $\mathbf{N}$ & $\mathbf{C M}$ & $\mathbf{N}$ & $\mathbf{C M}$ & $\mathbf{N}$ \\
\hline Leve & 06 & 02 & 01 & - & - & - & 06 & 02 & 06 & 01 & - & - \\
\hline Moderado & 06 & 04 & 01 & 01 & 01 & 01 & 06 & 02 & 03 & 02 & 02 & - \\
\hline Alto & 10 & 12 & 04 & 03 & 02 & 01 & 08 & 11 & 01 & 02 & 06 & 07 \\
\hline Total & \multicolumn{2}{|c|}{40} & 06 & 04 & 03 & 02 & 14 & 15 & 10 & 05 & 08 & 07 \\
\hline
\end{tabular}

*Clínica M édica

**N eurologia Clínica e Cirúrgica

levado ao desenvolvimento de UP em calcâneo $E$.

Em Risco A lto na N eurologia Clínica e Cirúrgica havia 12 pacientes: a) 03 foram admitidos com UP. b) 01 paciente desenvolveu U.P. ao longo do internamento apresentando Tumor M edular e como co-morbidades HAS. Estava sonolento, pouco comunicativo, poliqueixoso, sem movimentação ef etiva no leito esem acompanhante.

Em síntese os dados encontrados neste estudo mostraram que dentre os 40 pacientes pesquisados, 10 pacientes foram admitidos com UP com idade que variou de 21 a 92 anos, com predomínio da idade acima de 60 anos (07 pacientes). Houve 05 casos de pacientes que desenvolveram UP entre 8 e 26 dias de internamento. Dentre esses, a idade variou de 28 a 79 anos, com predomínio da idade acima de 60 anos (03 pacientes).

As patologias mais comuns nesse grupo de pacientes foram: Pneumonia, Doença Pulmonar Obstrutiva Crônica (DPOC), Tumor medular e cerebral, M ielopatia, Encefalopatia A Icoólica, Pielonefrite e A cidente Vascular Encefálico (AVE). A ssociadas a AVE, Diabetes M ellitus (DM) e Hipertensão A rterial Sistêmica (HAS).

U $m$ fato relevante obtido por intermédio de diálogos informais com auxiliares de enfermagem, é a dificuldade de perceber as úlceras de pressão em estágio I, ou seja, a hiperemia local que não fica pálida ao toque ${ }^{(12)}$.

Os estágios I e II podem passar despercebidos, mas na realidade, são sinais indicativos de isquemia em profundidade e são nesses estágios que as medidas de prevenção devem acontecer, com atuação efetiva da enfermagem. No entanto, as UP, normalmente, são diagnosticadas nos estágios III e IV, quando já existe necrose que necessita de tratamento local conservador ou até de tratamento cirúrgico. Portanto, enfatiza-se a importância das medidas preventivas nos estágios iniciais das UP(8)

A lgumas diretrizes neste sentido preconizam que os indivíduos considerados predisponentes ao desenvolvimento da UP devem ser reposicionados no leito pelo menos a cada duas horas, os horários devem ser pré-estabelecidos, estando disponíveis por escrito para que sejam realizadas as mudanças de decúbito(11).

$\mathrm{Na}$ mesma literatura encontram-se outras recomendações como: para minimizar as forças de cisal hamento e fricção, não se deve arrastar o paciente durante transferências no leito ou em mudanças de decúbito; deve-se manter a pele limpa, livre de umidade com o auxílio de fraldas descartáveis, forros impermeáveis à perspiração, ou, também, com o uso de cremes ou óleos que promovam barreira contra a umidade e hidratação adequada a fim de evitar ressecamentos. D estaca-se que todas as intervenções e resultados obtidos através desses cuidados devem ser acompanhados e documentados no prontuário, mediante prévia avaliação do seu estado geral, com o exame físico e do estado psicossocial(11).

Evidencia-se nas unidades estudadas que as equipes de enfermagem não fazem uso regular de instrumentos que poderiam auxiliar mais na melhoria da qualidade da assistência de enfermagem prestada, tal como a Escala de Braden e o SCP, as quais auxiliam a recuperar a saúde dos indivíduos hospitalizados e evitam complicações advindas do internamento e, dentre essas, a UP. 


\section{CONSIDERAÇÕES FINAIS}

A pós a experiência vivenciada durante a coleta de dados e sua análise, pode-se realizar algumas considerações em relação aos instrumentos de coleta de dados: SCP e Escala de Braden.

O SCP favorece a racionalização de recursos materiais e humanos. Possibilita ao enfermeiro conhecer 0 grau de dependência dos pacientes em relação aos cuidados de enfermagem considerando a sua individualidade, aspectos psicossociais e a promoção de ações educativas.

Embora 0 instrumento considere 0 acompanhante como membro importante no auxílio de al guns cuidados ao paciente e para a compreensão de al gumas informações para a reabilitação, por meio da Educação em Saúde, a maior parte dos acompanhantes da clientela presente nas unidades pesquisadas não atendeu às expectativas contidas no instrumento, por diversos fatores, entre eles cognitivos, sociais e educacionais.

A Escala de B raden contemplou os fatores que predispõem os indivíduos hospitalizados ao desenvolvimento das UP, podendo ser utilizada a todos os pacientes acamados, pois sua aplicação é fácil e dinâmica.

Outras considerações são feitas em relação à rotina do cuidado de enfermagem relacionado à prevenção e tratamento das úlceras de pressão, contemplando vários aspectos envolvidos nesse processo. Dentre eles destaca-se: durante o estudo observou-se a real ização dos curativos nas UP pelos auxiliares de enfermagem, com decisão do tratamento feita pelas enfermeiras da Comissão de Curativo do hospital. Observou-se, também, que pacientes, com UP gerada em internamentos anteriores, têm grande dificuldade de promover a cicatrização fora do ambiente hospitalar, e muitas vezes é re-internando por lesões infectadas e com áreas de necrose, fazendo uso então, de antibióticoterapia.

Evidenciamos vários fatores de risco para 0 desenvolvimento das UP, como fatores externos e internos ao paciente, como já relatamos na revisão de literatura. Complementar a esses, encontramos fatores relacionados à estrutura da instituição de saúde, dentre eles estão a fal ta de condições para a implementação e manutenção de medidas preventivas, bem como 0 número insuficiente de profissionais e o preparo desses para realizar o cuidado.

As medidas preventivas requerem esforços da instituição, principalmente, financeiros. Para a sua implementação é preciso investir em cuidados especializados, em materiais adequados para 0 alívio da pressão nas regiões de proeminências ósseas, pois a pressão é o fator externo mais evidente na formação das UP(13).

Enfim, a prevenção das úlceras de pressão deve ser feita por meio de programas educacionais estruturados, organizados, amplos, direcionados a profissionais de saúde, cuidadores, pacientes e familiares ${ }^{(11)}$.

A creditamos que as UP podem se prevenidas, mas, para tanto, é necessário o envolvimento da instituição, dos profissionais de saúde e, dentre esses, da equipe de enfermagem. Destes últimos, acreditamos que o processo educacional é primordial para o fim proposto. Tal afirmativa é reforçada em estudo sobre a incidência de úlceras por pressão em um hospital universitário, indicando a urgente necessi dade da implantação de programa de prevenção e tratamento de UP em instituições ${ }^{(14)}$.

\section{REFERÊNCIAS}

1. Carmona L M P, Évora Y D M. Sistema de classificação de pacientes: aplicação de um instrumento validado. Rev Escol Enferm USP 2002; 36(1):42-9.

2. Smeltzer $S C, B$ are BG, Brunner e Suddarth: Tratado de enfermagem médico-cirúrgica. Rio de Janeiro: Guanabara K oogan; 2002.

3. Blanes L, Duarte IS, Calil J A, Ferreira L M . Avaliação clínica e epidemiológica das úlceras por pressão em pacientes internados no Hospital São Paulo. Rev A ssoc M ed B ras 2004; 50(2):182-7.

4. Hess CT. Enfermagem prática: Tratamento de feridas eúlceras. Rio deJ aneiro: Reichmann eA ffonso; 2002.

5. Perroca M G, Gaidzinski RR. Sistema de classificação de pacientes: construção e validação de um instrumento. Rev Escol Enferm USP 1998; 32(2):153-68.

6. Paranhos WY. Úlceras de pressão. In: J orgeA S, Dantas SR PE. A bordagem multiprofissional do tratamento de feridas. São Paulo: A theneu; 2003.

7. Dealy C. Cuidando de feridas: um guia para as enfermeiras. São Paulo: A theneu; 2001.

8. Ferreira LM , Calil JA. Etiopatogenia e tratamento das úlceras por pressão. Rev Diag Trat 2001; 6(3): 36-40.

9. Fernandes $L M, B$ raz E. Avaliação de risco para o desenvolvimento de úlceras de pressão em pacientes internados em centro de terapia intensiva. Cogitare 
Enferm 2002; 7(1):17-22.

10. B ergstrom $N$. The $B$ raden scale for predicting pressure sore risk. Nurs. Res 1987; 36(4):205-10.

11. Rangel EML. Conhecimento, práticas e fontes de informação de enfermeiros de um hospital sobre a prevenção e tratamento da úlcera de pressão [dissertação]. Ribeirão Preto (SP): Escola de Enfermagem de Ribeirão Preto USP; 2004.

12. Lueckenotte, A. Enfermagem prática: Avaliação em gerontologia. Rio de Janeiro: Reichmann e Affonso; 2002.

13. Fernandes $L M$. Úlceras de pressão em pacientes críticos hospital izados - uma revisão integrativa da literatura [dissertação]. Ribeirão Preto (SP): Escola de Enfermagem de Ribeirão Preto USP; 2000.

14. Rogenski NM B, Santos VLCG. Estudo sobre a incidência de úlceras por pressão em um hospital universitário. Rev. Latino-am. Enferm 2005; 13(4):47480. 